\title{
Impact of Global Warming on Public Health
}

\author{
Magaille Hodambia ${ }^{1}$, Sindi Dandala ${ }^{1}$ \\ ${ }^{1}$ Faculty of Health Sciences, University of Cape Town, South Africa
}

\begin{abstract}
This article aims to explain the impact of global warming on public health. The mechanism that occurs is that climate change affects environmental factors such as changes in environmental quality such as water quality, air and soil, depletion of the ozone layer, decreases in the quality and quantity of water resources, loss of ecosystem function and land degradation which in the end these factors will affect human health. . Global warming has shortened the mating cycle and growth of mosquitoes from eggs to larvae and adult mosquitoes, so that the population size will increase rapidly. The impact of global warming also affects ozone depletion, among others, the increasing intensity of ultraviolet rays reaching the earth's surface causing health problems, such as skin cancer, cataracts, decreased endurance, and the growth of genetic mutations. . It is also associated with high air temperature with decreased heart rate. A low heart rate can increase the risk of a heart attack.
\end{abstract}

Keywords: Global Warming, Public Health, Environmental Problems

\section{Introduction}

The African continent is one of the countries with the highest levels of biodiversity in the world. Forests and seas in this country, including the richest ecosystems in the world, provide employment and income to millions of inhabitants of the African continent. However, the environment of this country is under intense stress due to human activities. The exploitation of natural resources is an important part of the country's economy as a result of which various sectors related to natural resource management, such as the forestry sector, are in a dire state because their resources continue to run low (Daniele, 2011; Nelsons \& Agrawal, 2008; Stijns, 2005; Broad, 1995).

Global warming has caused significant climate change, as happened in our country, the effect of this warming has caused extreme climate change. In some areas, heavy rains often occur which cause flash floods and landslides, the appearance of tornado winds, and even drought that threatens human life (Ghosh, 2018; Middleton \& Sternberg, 2013; Mooney, 2007; Kadomura, 2005). This paper will discuss the definition of global warming, the result of global warming, how to prevent global warming, measure global warming and the major disasters caused by global warming.

As we know, all energy sources on Earth come from the Sun. Most of this energy is in the form of short wave radiation, including visible light. When this energy hits the surface of the Earth, it changes from light to heat which warms the Earth. Earth's surface, will absorb some of the heat and reflect the rest back as long-wave infrared radiation to outer space. However, some of the heat remains trapped in the earth's atmosphere due to the accumulated amount of greenhouse gases that trap these radiation waves. 
In general, global warming is defined by increasing the temperature of the earth's surface by greenhouse gases due to human activities. Although local temperatures fluctuate naturally, in the last 50 years global temperatures have tended to increase more rapidly than previously recorded data. And the ten warmest years occurred after 1990. The issue of global warming has been growing recently. The main character is, of course, a human with various activities.

The mechanism that occurs is that climate change affects environmental factors such as changes in environmental quality (water quality, air and soil), depletion of the ozone layer, decreases in the quality and quantity of water resources, loss of ecosystem function and land degradation which in the end these factors will affect health. humans and the environment. The close relationship between climate and its change on health, makes me interested in discussing more about the impact of Global Warming on Health.

\section{Understanding Global Warming}

Maybe you've imagined being in a tightly closed car during the day. Sunlight can freely enter the car through the windshield, causing the air in the car to become warmer. The air in the car warms up, because the heat of the incoming sunlight cannot freely go out. So that the heat is trapped in the car.

Likewise with global warming. The sun radiates to Earth through the layers of the earth's atmosphere. This radiation will be reflected back into space, but some of the waves are absorbed by greenhouse gases, namely CO2, ch4, N2O, HFCs, and SF4 which are in the atmosphere. As a result these waves are trapped in the earth's atmosphere. These events occur repeatedly, causing the average temperature at the earth's surface to increase. This event is often referred to as global warming.

Global warming is an increase in the average temperature of the earth's surface due to an increase in the amount of greenhouse gas emissions in the atmosphere. Global warming will be followed by climatic changes, such as increased rainfall in several parts of the world, causing flooding and erosion (Solomon et al, 2009). Meanwhile, other parts of the earth will experience a prolonged dry season due to rising temperatures.

\section{Global Warming by Greenhouse Gases}

Greenhouse gases 『(CO _ (2,) 『CO $\rrbracket_{-}($, ) N_2 O) produced by industrial combustion residues will have an effect like a greenhouse, where indoors is hotter than outdoors, this is because heat entering the room is radiation trapped by the glass layer.

At first, the energy of sunlight is radiated to the earth, the sun's rays are radiated in the form of high energy rays (UV), visible light (VIS), and low energy (IR). The light radiation will then reach the ground and part of it will be reflected (low energy rays), some will be absorbed (high energy rays and visible rays). Earth has a protective layer called the atmosphere, the most important part of the artmosphere to protect from UV radiation is O_3 (Ozone), high energy rays will be converted into rays with a longer wavelength, namely in the form of Infra Red.

Visible and infrared rays of the sun will reach the surface of the earth, then some are absorbed, partially reflected, more IR radiation is reflected than Vissible rays. The reflected IR radiation will return to the atmosphere, as a result of the accumulation of $\mathrm{CO} 2$ and other greenhouse gases, this IR light radiation is trapped, because the greenhouse gas layer has the property of being impenetrable by long wave radiation (IR). As a result, IR radiation will be trapped in the

Copyright (C) 2020, Journal Wetenskap Health, Under the license CC BY-SA 4.0 
troposphere which then results in an accumulation of heat energy, this accumulation of heat energy which causes the earth's surface temperature to continue to rise. Which was later called Global Warming.

\section{Impact of Global Warming on Healths}

The dangers of climate change in the continent of Africa for the future of health are characterized by a significant increase in rainfall in certain months with increased variability in certain areas, a decrease in rainfall in dry months, while in the wet season the rainfall increases, increase in average surface temperature.

The dangers of climate change affect health through microbial contamination pathways and dynamic transmission. Health impacts that can occur from this process include the effects of increasing temperatures on morbidity and mortality, disasters due to extreme weather, increased air pollution, water and foodborne diseases, and vector and rodent-borne diseases.

\section{Diarrhea \& Malnutrition}

Climate change is causing sea levels to rise due to melting polar ice, damaging mangrove ecosystems, causing sea water intrusion into land, making fresh water increasingly difficult to obtain, and narrowing land used for agriculture (Mimura, 2013). Increasing sea levels also cause flooding that destroys food crops. The existence of prolonged drought causes drought, and these factors greatly support the occurrence of crop failure, so that food availability decreases and at the household level the nutritional intake also decreases. Low nutritional intake and difficulty in obtaining clean water and sanitation facilities lead to infectious diseases that exacerbate malnutrition. From the marine sector, an increase in sea water temperature and the presence of sea storms reduce fish production which affects the availability of fish as food, affects nutritional intake in the household and exacerbates the occurrence of malnutrition (De Silva \& Soto, 2009).

The occurrence of malnutrition causes the death of 3.7 million people per year and diarrhea results in the death of 1.9 million people, Drought results in a decrease in the nutritional status of the community due to disrupted harvests, floods cause widespread diarrhea and leptospirosis (a disease carried by rodents).

\section{Acute Respiratory Infection (ARI)}

Forest fires, can disrupt the earth's ecosystem, produce greenhouse gases that cause global warming. While the black smoke directly disturbs human life, the smoke which contains fine dust and various carbon oxides causes respiratory problems and acute respiratory infections (ARI), ranging from asthma, bronchitis to chronic obstructive pulmonary disease (COPD). The smoke also carries dioxin toxins which can cause lung cancer and pregnancy problems and infertility in women.

\section{Dengue Fever $(\mathrm{DHF})$ \& Malaria}

Ecosystem displacement can have an impact on the spread of water-borne diseases and vectorborne diseases. Such as the increasing incidence of Dengue Fever due to the emergence of a new space (ecosystem) for these mosquitoes to breed. With this climate change, there are several species of disease vectors (eq Aedes Agipty), viruses, bacteria, plasmodium becoming more resistant to certain drugs that target these organisms. DHF itself is related to the erratic rainy season. Another impact that can be felt is that mosquitoes are increasingly breeding, especially in Africa and Asia. Two serious diseases caused by mosquito bites, namely malaria and dengue

Copyright (C) 2020, Journal Wetenskap Health, Under the license CC BY-SA 4.0 
hemorrhagic fever, are very sensitive to climate change. On the African continent we have experienced it firsthand, namely the high number of victims suffering from dengue fever.

Global warming has shortened the mating cycle and growth of mosquitoes from eggs to larvae and adult mosquitoes, so that the population size will increase rapidly. Regarding the relationship between global warming and the increase in dengue fever vectors, it can be explained as follows: Hot and humid air is most suitable for the malaria mosquito (Anopheles), and the dengue mosquito (Aedes aegypti).

In the past, these two types of killer mosquitoes appeared more frequently in the transition season, the transition between the rainy and dry seasons. Now the time span of the attacks of the two insects is almost all year round. Hot and humid air lasts all year round, coupled with poor sanitation which always provides clear puddles for them to lay their eggs. So, now the malaria virus carried by Anopheles and the dengue virus carried by the Aedes aegypti mosquito can attack at any time viciously.

Due to global warming, the extrinsic incubation cycle of the virus that causes dengue hemorrhagic fever (DHF) in the Aedes aegyti mosquito and the extrinsic incubation cycle of the virus that causes malaria in the Anopheles mosquito is shorter and the incubation period for germs is shorter (Paaijmans et al., 2009; Bellan, 2010). Their population is more explosive. As a result, cases of dengue fever have increased more easily than in previous years. The highest number of deaths currently occurs in children. Data from the World Health Organization (WHO) in 2010 noted that the incidence of dengue fever has increased over the last 50 years. These incidents occur in both tropical and sub-tropical urban areas, attacking more than 100 million people each year. Then, about 30 thousand deaths occurred in children.

\section{Degenerative disease}

Global warming makes the air temperature hotter, which can cause additional pollution. This increase in pollution levels has a bad effect on the heart. In addition, research has also shown that higher temperatures and ozone damage can make heart health worse. This is associated with high air temperature with a decrease in heart rate. A low heart rate can increase the risk of a heart attack. Researchers also say higher temperatures can make the body more sensitive to toxins.

\section{Diseases due to thinning of the ozone layer}

The impact of global warming also affects ozone depletion, among others, the increasing intensity of ultraviolet rays reaching the earth's surface causing health problems, such as skin cancer, cataracts, decreased endurance, and the growth of genetic mutations.

The impact of global warming that occurs in each country is different due to the fact that the climate in each country is different, namely consisting of tropical and subtropical. In subtropical countries that have 4 seasons, the impact of global warming mainly occurs in temperature changes that are getting more extreme during summer (warmer temperatures) and during winter (cooler temperatures). Meanwhile, the impact that occurs in the tropics mainly affects the shifting of seasons (beginning and end of the rainy or dry season) and the increase in cases of disease outbreaks. In addition, the impact felt by island countries is the threat of reducing the length of the coastline due to rising sea levels due to melting of the polar ice caps.

The impact that occurs due to global warming is very diverse, namely the impact on weather, sea level, agriculture, animals and plants and human health. Due to global warming, the

Copyright (C) 2020, Journal Wetenskap Health, Under the license CC BY-SA 4.0 
temperature in winter and at night will tend to increase. Rainfall increases, water will evaporate more quickly from the ground, as a result, some areas will become drier than before. Hurricanes are more common. "Global warming will melt a lot of ice at the poles. As a result, sea levels around the world have increased by 4-8 inches over the past 100 years. Yet according to calculations by IPCC experts, a $100 \mathrm{~cm}$ increase in sea level will drown 6 percent of the Netherlands, 17.5 percent of Bangladesh. And thousands of small islands in the African continent will sink".

With this fact, it is clear that Global Warming has a major impact on the environment and the surface of the earth as a place for humans and other living creatures to live. In essence, global warming can not be avoided, but it can be reduced if only we know the causes and try to reduce the bad effects it causes.

\section{How to Minimize Global Warming}

Scientists study ways to limit global warming. The main key is: limiting CO2 emissions. There are two effective techniques for limiting carbon emissions, namely replacing oil energy with other energy sources that do not emit carbon and secondly using oil energy as economically as possible.

Alternative energies that can be used include wind, sunlight, nuclear energy, and geothermal energy. Windmills can convert wind energy into electrical energy. Sunlight can also be converted into electrical energy or heat sources that can be used such as water heaters, solar stoves, etc. Geothermal energy can be used to generate electricity.

Alternative energy sources are indeed more expensive than oil energy, but further research will help to further reduce costs. $\mathrm{CO} 2$ emissions could be reduced if cars could be more fuel efficient. Scientists and engineers have worked to create engines that are fuel efficient. Inventions have developed means to replace combustion engines or use smaller engines. A battery-electric car has entered the market, but is still equipped with a small, oil-fired engine.

Fuel cells believe a device capable of converting chemical energy into electrical energy can be developed for cars in the future. Hiding carbon also helps prevent carbon dioxide from entering the atmosphere or taking up existing CO2. Hiding carbon can be done in 2 ways, namely underground or groundwater storage and storage in living plants.

\section{Conclusion}

Global warming is the process of increasing the average temperature of the atmosphere, sea, and earth's land. Part of this heat is as long-wave infrared radiation into outer space. The increase in global temperature is expected to cause other changes such as an increase in the intensity of extreme weather phenomena. Global warming is the result of human activities that tend to be possibleistik humans can change nature. The impact of global warming also affects ozone depletion, among others, the increasing intensity of ultraviolet rays reaching the earth's surface causing health problems, such as skin cancer, cataracts, decreased endurance, and the growth of genetic mutations.

\section{References}

Bellan, S. E. (2010). The importance of age dependent mortality and the extrinsic incubation period in models of mosquito-borne disease transmission and control. PLoS One, 5(4), e10165. 
Broad, R. (1995). The political economy of natural resources: Case studies of the Indonesian and Philippine forest sectors. The Journal of Developing Areas, 29(3), 317-340.

Daniele, V. (2011). Natural resources and the 'quality'of economic development. the Journal of Development studies, 47(4), 545-573.

De Silva, S. S., \& Soto, D. (2009). Climate change and aquaculture: potential impacts, adaptation and mitigation. Climate change implications for fisheries and aquaculture: overview of current scientific knowledge. FAO Fisheries and Aquaculture Technical Paper, 530, 151212.

Ghosh, A. (2018). The great derangement: Climate change and the unthinkable. Penguin UK.

Kadomura, H. (2005). Climate anomalies and extreme events in Africa in 2003, including heavy rains and floods that occurred during northern hemisphere summer.

Middleton, N. J., \& Sternberg, T. (2013). Climate hazards in drylands: A review. Earth-Science Reviews, 126, 48-57.

Mimura, N. (2013). Sea-level rise caused by climate change and its implications for society. Proceedings of the Japan Academy, Series B, 89(7), 281-301.

Mooney, C. C. (2007). Storm world: Hurricanes, politics, and the battle over global warming. Houghton Mifflin Harcourt.

Nelson, F., \& Agrawal, A. (2008). Patronage or participation? Community-based natural resource management reform in sub-Saharan Africa. Development and change, 39(4), 557-585.

Paaijmans, K. P., Read, A. F., \& Thomas, M. B. (2009). Understanding the link between malaria risk and climate. Proceedings of the National Academy of Sciences, 106(33), 1384413849.

Solomon, S., Plattner, G. K., Knutti, R., \& Friedlingstein, P. (2009). Irreversible climate change due to carbon dioxide emissions. Proceedings of the national academy of sciences, 106(6), 1704-1709.

Stijns, J. P. C. (2005). Natural resource abundance and economic growth revisited. Resources policy, 30(2), 107-130. 